\title{
FLNA is implicated in pulmonary neuroendocrine tumors aggressiveness and progression
}

\author{
Eleonora Vitali ${ }^{1}$, Ilena Boemi ${ }^{1}$, Lorenzo Rosso ${ }^{2}$, Valeria Cambiaghi', Pierluigi \\ Novellis ${ }^{3}$, Giovanna Mantovani ${ }^{4}$, Anna Spada4, Marco Alloisio ${ }^{3,5}$, Giulia Veronesi ${ }^{3}$, \\ Stefano Ferrero ${ }^{6}$ and Andrea G. Lania ${ }^{1,7}$

\footnotetext{
${ }^{1}$ Laboratory of Cellular and Molecular Endocrinology, IRCCS Clinical and Research Institute Humanitas, Milan, Italy

${ }^{2}$ Thoracic Surgery and Lung Transplantation Unit, Fondazione IRCCS Ca' Granda Ospedale Maggiore Policlinico, Milan, Italy

${ }^{3}$ Humanitas Clinical and Research Center, Thoracic Surgery Division, Milan, Italy

${ }^{4}$ Fondazione IRCCS Ospedale Maggiore Policlinico, Endocrinology and Diabetology Unit, Department of Clinical Sciences and Community Health, University of Milan, Milan, Italy

${ }^{5}$ Department of Biomedical Sciences, Humanitas University, Milan, Italy

${ }^{6}$ Division of Pathology, Fondazione IRCCS Ca' Granda Ospedale Maggiore Policlinico, Milan, Italy

${ }^{7}$ Endocrinology Unit, Department of Biomedical Sciences, Humanitas University and Humanitas Research Hospital, Milan, Italy
}

Correspondence to: Eleonora Vitali, email: Eleonora.vitali@humanitasresearch.it

Keywords: pulmonary neuroendocrine tumors, Filamin A, Rapl GTPase, cell migration, cell proliferation

Received: March 29, $2017 \quad$ Accepted: July 25, $2017 \quad$ Published: August 24, 2017

Copyright: Vitali et al. This is an open-access article distributed under the terms of the Creative Commons Attribution License 3.0 (CC BY 3.0), which permits unrestricted use, distribution, and reproduction in any medium, provided the original author and source are credited.

\section{ABSTRACT}

Pulmonary neuroendocrine tumors (PNTs) comprise different neoplasms, ranging from low grade carcinoids to the highly malignant small cell lung cancers. Several studies identified the cytoskeleton protein Filamin A (FLNA) as determinant in cancer progression and metastasis, but the role of FLNA in PNT aggressiveness and progression is still unknown.

We evaluated FLNA expression in PNTs with different grade of differentiation, the role of FLNA in cell proliferation, colony formation, angiogenesis, cell adhesion and migration in PNT cell line (H727 cells) and primary cultures and the possible interaction between FLNA and Rap1-GTPase. FLNA is highly expressed in PNTs with high malignant grade. FLNA silencing reduces cyclin D1 levels $(-51 \pm 5, p<0.001)$ and cell proliferation in PNT cells $(-37 \pm 4, p<0.05)$, colony formation and VEGF expression $(-39 \pm 9 \%, \mathrm{p}<0.01)$ in $\mathrm{H727}$ cells.

FLNA and Rap1 co-localize in cellular protrusions and FLNA silencing up-regulates Rap1 expression $(+73 \pm 18 \%, p<0.01)$.

Rap1 silencing prevents cell adhesion increase $(+43 \% \pm 18 \%, p<0.01)$ and cell migration decrease $(-56 \pm 7 \%, \mathrm{p}<0.01)$ induced by FLNA silencing, without affecting cell proliferation reduction. In conclusion, FLNA is implicated in PNT progression, in part through Rap1, thus providing a potential diagnostic and therapeutic target.

\section{INTRODUCTION}

Pulmonary neuroendocrine tumors (PNTs) comprise a spectrum of neoplasms, ranging from low grade typical carcinoids via the intermediate grade atypical carcinoids to the highly malignant small cell lung cancers [1]. Since no large phase II/III trials for PNTs have been published, surgery remains the treatment of choice $[2$, 3]. Different studies clearly support a role of a widelyexpressed cytoskeleton protein, Filamin-A (FLNA), in cancer progression and metastasis [4]. In fact, FLNA is overexpressed in multiple types of tumors, including prostate, breast, lung cancer, hemangiomas, colon cancer, melanoma, neuroblastoma, squamous cell carcinoma, 
hepatic cholangiocarcinoma, suggesting a possible correlation with FLNA and cancer aggressiveness $[5,6]$.

FLNA is crucial for cell shape modulation and motility: it crosslinks cortical actin filaments into a dynamic three-dimensional structure [7] and anchors actin filaments to cell-extracellular matrix adhesion sites. Moreover, FLNA is known to scaffold over 90 protein-binding partners, involved in receptor activation, cell migration and adhesion, cell proliferation, inflammation and tumorigenesis [8-10]. Increasing evidence demonstrated that FLNA is pivotal for regulation of matrix cytoskeleton signaling by binding to integrins, which are essential for maintaining directed cell migration and adhesion [6], and for coordination of GTPase signaling factors, leading the formation of lamellipodia and filopodia [11]. In this respect, Rap1 small GTPase is involved in the control of cell migration and adhesion by enhancing integrin-mediated cell-matrix attachment in several cell types [12].

In addition, Filamin-A (FLNA) physically interacts with HIF-1 $\alpha$ [13], that regulates angiogenesis through upregulation of vascular endothelial growth factor (VEGF) $[14,15]$.

To date, the role of FLNA and the molecular mechanism involved in PNT aggressiveness and progression is still unknown. In order to establish whether FLNA is involved or not in this phenomenon we evaluated the correlation of FLNA expression patterns in different PNT stages, we assessed the role of this cytoskeleton protein in influencing cell mobility, cell proliferation, colony formation, angiogenesis, focusing on the potential interaction between FLNA and Rap1.

A)

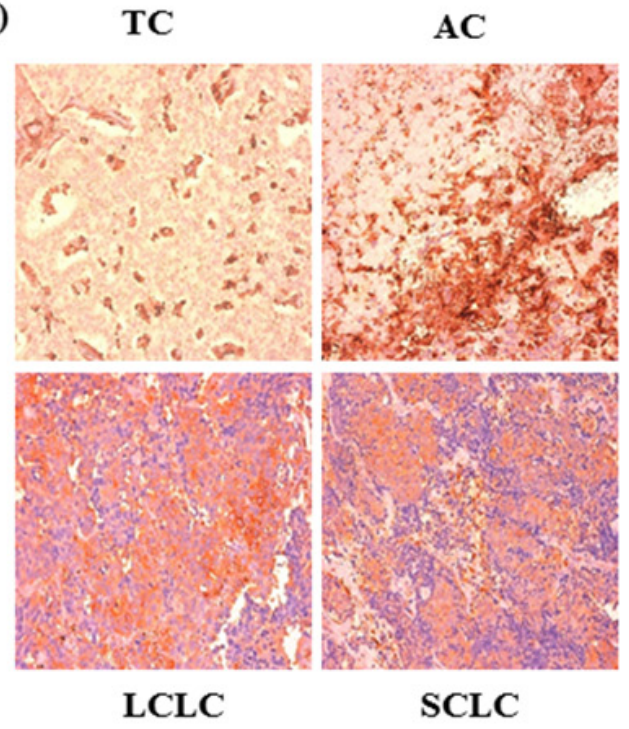

\section{RESULTS}

\section{Filamin A expression correlates with different PNTs stages}

In order to study the possible relationship between FLNA and tumor aggressiveness, we evaluated FLNA expression in different PNT stages by immunohistochemistry using PNT samples, ranging from low grade typical carcinoids (TP) $(\mathrm{n}=40)$, atypical carcinoids (AT) $(\mathrm{n}=11)$ to the highly malignant large cell lung carcinoma ( $\mathrm{LCLC})(\mathrm{n}=10)$ and small cell lung cancers $(\mathrm{SCLC})(\mathrm{n}=5)$. We found a correlation between clinical phenotype of different P-NETs and immunohistochemical score of FLNA. As shown in Figure 1A FLNA is expressed in the cytoplasm of PNT tissues and its expression being higher in PNTs with higher malignant grade. In particular, FLNA expression is significantly increased in LCLC and SCLC cells compared to TC and AC cells (one way ANOVA, followed by Bonferroni post hoc test, **** $\mathrm{p}<0.001, * * \mathrm{p}<0.01$ ) (Figure 1B). As shown in Table 1, FLNA expression is significantly correlated with age, gender, $\mathrm{T}$ and $\mathrm{N}$ staging $(* \mathrm{p}<0.05 ; * * \mathrm{p}<0.01)$. The pathological findings of 65 PNTs included in the IHC study are described in the Supplementary Table 1.

\section{FLNA promotes PNTs cell proliferation and colony formation}

To further investigate the role of FLNA in tumor development and progression, we evaluated whether
B)

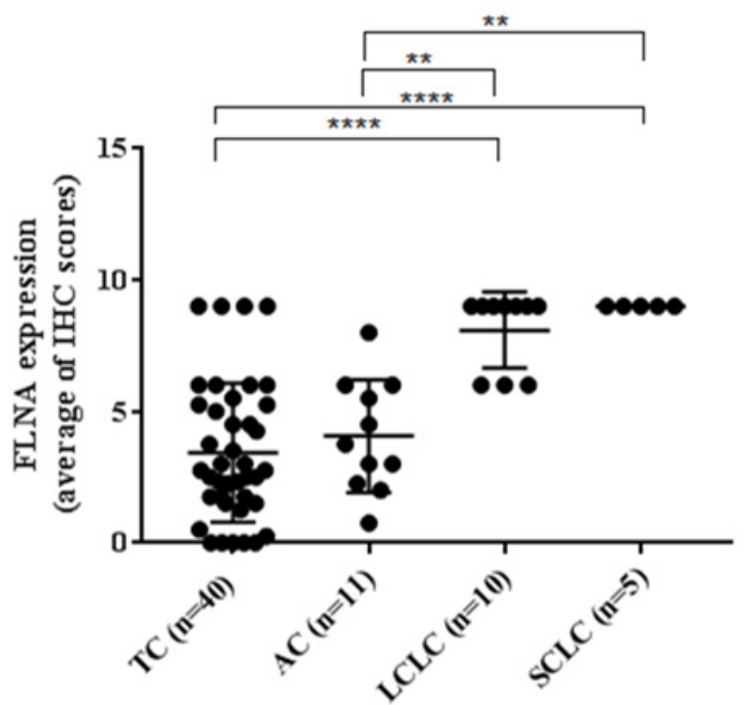

Figure 1: (A) Representative pictures of immunohistochemistry for FLNA in different PNTs (20X magnification). (B) Graph of FLNA expression obtained from the average of immunohistochemistry scores in different PNTs. FLNA immunoreactivity was graded taking into account both the percentage of positive cells $(0-30 \%=1 ; 31-60 \%=2 ; 61-100 \%=3)$ and the staining intensity $(0=$ absence of immunoreactivity; $1=$ weak; $2=$ medium intensity; and $3=$ strong reactivity). $\mathrm{TC}=$ typical carcinoid, $\mathrm{AC}=$ atypical carcinoid, $\mathrm{LCLC}=$ large cell lung carcinoma; SCLC $=$ small cell lung carcinoma. 
Table 1: Associations between FLNA expression and clinicopathological features in PNTs

\begin{tabular}{lcc}
\hline Factor & FLNA H scores (average) & P-value \\
\hline Age (years) & & $* * \mathrm{a}$ \\
$\leq 50$ & 3,06547619 & \\
$>50$ & 5,376539142 & $* * \mathrm{a}$ \\
Gender & & \\
M & 6,021481481 & \\
F & 4,032439024 & \\
T & & \\
T1 & 4,321891892 & \\
T2 & 4,95 & \\
T3 & 6,625 & \\
T4 & 9 & \\
N & & \\
N0 & 4,229038462 & \\
N1 & 5,125 & \\
N2 & 7,178571429 & \\
\hline
\end{tabular}

The association was identified with the appropriated statistical test, t-test or ANOVA when required.

F, female; M, male.

${ }^{\mathrm{a}}$ t-test; ${ }^{\mathrm{b}}$ ANOVA.

$\mathrm{T}$, primary tumor; $\mathrm{N}$, lymph node involvement.

FLNA is involved in PNT cell proliferation. Thus, we silenced FLNA expression in PNTs to evaluate cyclin D1 expression. As shown, 80\% decrease in FLNA levels significantly reduced cyclin D1 levels, with respect to PNT cells transfected with negative control (C- siRNA) $(-51 \pm 5$ $\mathrm{p}<0.001$ ) (Figure 2A).

To confirm these data, we evaluated cell proliferation by BrdU incorporation during DNA synthesis. FLNA silencing significantly decreased cell proliferation in primary PNT cells $(-37 \pm 4, \mathrm{p}<0.05$ vs C-siRNA (Figure 2B). These data suggest that FLNA promotes cell proliferation in PNTs.

To overcome the limitations due to the scarce availability and number of PNT cells, we used the human H727 cell line, as a model for typical carcinoids. To further investigate the promoting effects of FLNA in proliferation, we performed cell cycle analysis on $\mathrm{H} 727$ cells silenced for FLNA. As shown in Figure 2C FLNA knockdown significantly increased the percentage of $\mathrm{H} 727$ cells in the G1 phase and decreased in the S phase. Therefore, the cytostatic effect of FLNA silencing elicited by a decrease in cyclin D1 expression in PNTs cells is consistent with the inhibition of the cell cycle at the G1/S transition in H727 cell.

The tumorigenic properties of $\mathrm{H} 727$ cells transfected with C- siRNA or FLNA siRNA were compared by assessing their ability to grow in vitro and to form colonies.
As shown in Figure 2D, 7 days after plating, control H727 cells produced numerous colonies, whereas cells silenced for FLNA developed few and very small colonies. In addition, FLNA silencing significantly decreased the number of $\mathrm{H} 727$ cells $(-80 \pm 3 \%, \mathrm{p}<0.0001)$ and colony size (6.20 fold, $\mathrm{p}<0.001$ ) with respect to negative control cells (Figure 2E, 2F).

\section{FLNA is involved in angiogenesis increase in H727 cells}

The VEGF pathway, implicated in angiogenesis, has been demonstrated to be over-expressed in PNTs [14], thus we evaluated FLNA implication in angiogenesis through links with vascular endothelial growth factor (VEGF). As shown in Figure 3A,3B, VEGF expression decreased in primary PNT cells and H727 cells lacking FLNA $(-36 \pm 7 \%, \mathrm{p}<0.05$ vs C-siRNA and $-39 \pm 9 \%, \mathrm{p}<0.01$ vs C-siRNA, respectively). To confirm this result, we measured the levels of VEGF secreted by $\mathrm{H} 727$ cells transfected with C- or FLNA siRNA by ELISA. After 24 hours of culture VEGF release was drastically reduced in $\mathrm{H} 727$ cells transfected with FLNA siRNA compared to control, suggesting that FLNA silencing suppressed VEGF secretion. These data support the key role of FLNA in promoting angiogenesis. 


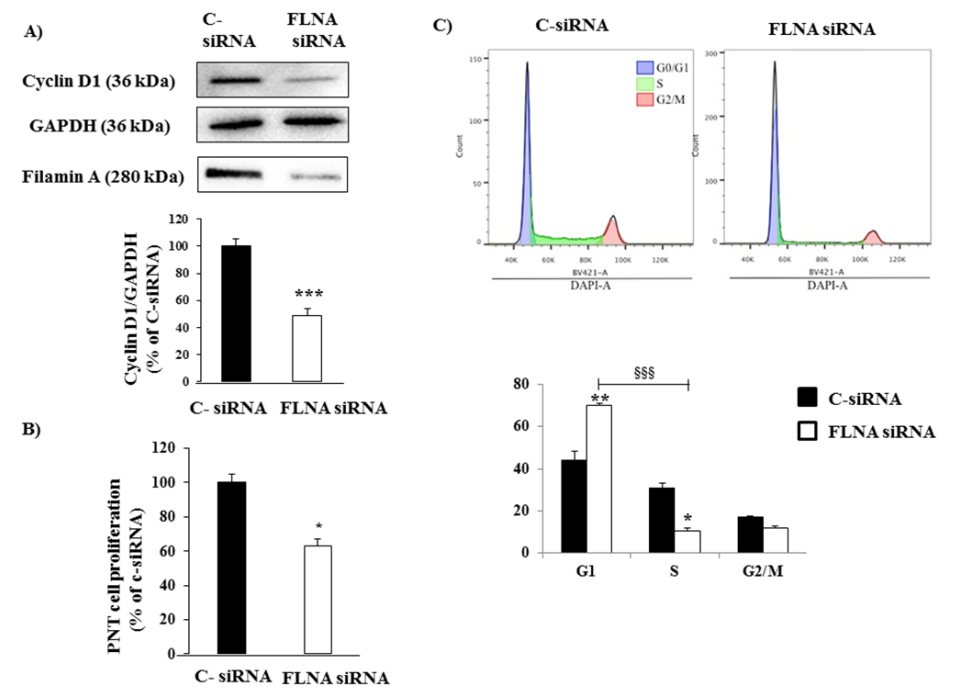

D)

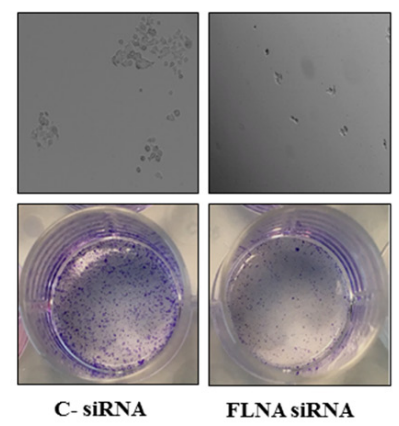

5)

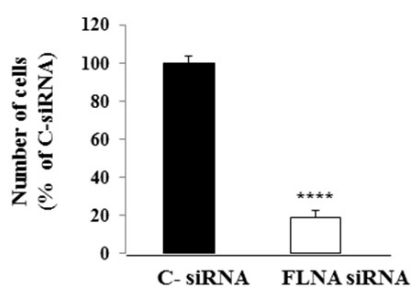

F)

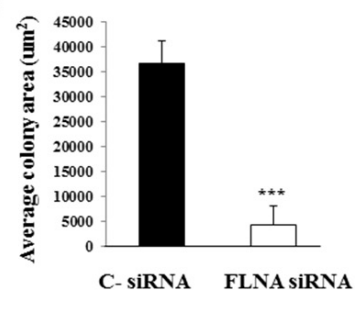

Figure 2: FLNA silencing reduced PNT cell proliferation and colony formation. (A) Representative immunoblotting performed with antibodies raised against Cyclin D1. FLNA knock down induced a significant decrease in Cyclin D1 levels in primary PNT cells. The equal amount of protein was confirmed by stripping and reprobing with an anti-GAPDH antibody. The graph shows the quantification of Cyclin D1 normalized to GAPDH. Data represent mean \pm SD of three independent experiments. $* * *=p<0.001$ vs C- siRNA. (B) After FLNA silencing, we measured cell proliferation in primary PNTs cells incubated with BrdU for $24 \mathrm{~h}$. Experiments were repeated 3 times and each determination was done in triplicate. Values represent mean $( \pm \mathrm{SD}) *=\mathrm{p}<0.05$ vs $\mathrm{C}$ - siRNA. (C) Cell cycle analysis by DNA content of H727 cells treated with C- siRNA or FLNA siRNA. H727 cell line were fixed and processed according to the DAPI labelling protocol listed on material and method. Single cells were gated via DAPI width and area signals to calculate G1, S phase, G2/M from a DAPI area histogram. Left panel represent $\mathrm{H} 727$ cells treated with C- siRNA, right panel represent H727 cells treated with FLNA siRNA. The graph shows the quantification of data from two independent experiments. Values represent mean $( \pm \mathrm{SD}) *=\mathrm{p}<0.05$, $* *$ $=\mathrm{p}<0.01, \S \S \S=0.001$. (D) FLNA silencing strongly decreased quantity of colonies. Microscopyimages (top panels) show H727 cells incubated with C-siRNA and FLNA siRNA after 7 days. Images of the cell colony assay stained with a solution containing $0.05 \%$ crystal violet (bottom panels). (E) To count numbers of cells, after 7 days H727 cells were incubated with cell quantification solution and absorbance was measured at $490 \mathrm{~nm}$. Graph shows that FLNA silencing significantly decreased number of $\mathrm{H} 727$ cells with respect to $\mathrm{C}$-siRNA cells. Values represent mean $\pm \mathrm{SD}$ of 3 experiments. $* * * *=\mathrm{p}<0.0001 \mathrm{vs}$ corresponding basal. (F) To measure colony surface area, 3-4 fields were randomly selected in each well. Average of colony area $(\mu \mathrm{m} 2)$ was measured using the software ImageJ. $* * *=\mathrm{p}<0.001$ vs corresponding basal. Experiments were repeated at least 3 times. Statistical analysis was performed with t-test. 


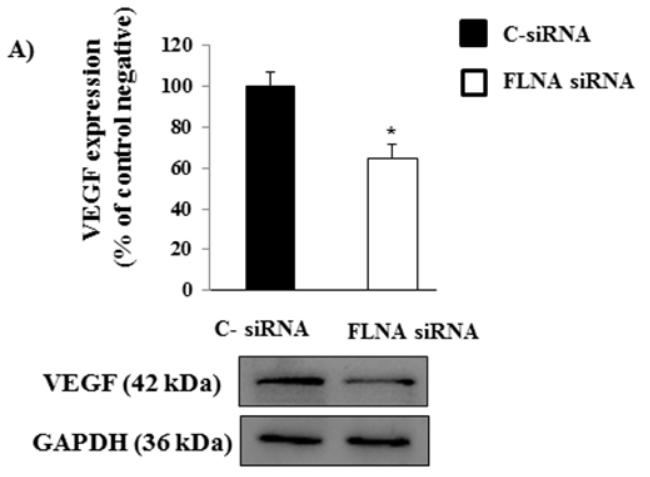

B)

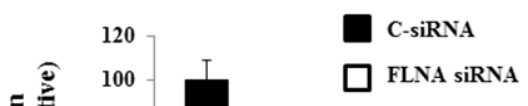

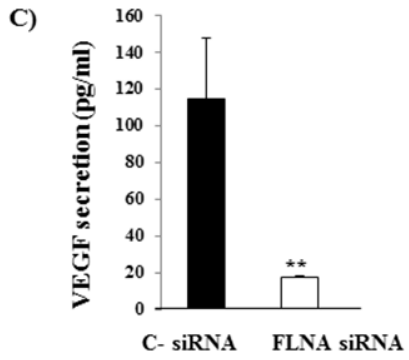

VEGF (42 kDa)

GAPDH (36 kDa)

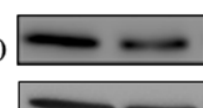

C- siRNA FLNA siRNA

Figure 3: Effect of FLNA on VEGF expression and in vitro release in $\mathbf{H 7 2 7}$ cells. (A\&B) Representative immunoblot of VEGF demonstrates that FLNA silencing reduces VEGF expression in primary PNTs cells and H727 cells, respectively. The graph shows the ratio of VEGF/GAPDH normalized to C- siRNA. Experiments were repeated at least 2-3 times. ${ }^{*} \mathrm{p}<0.05,{ }^{* *} \mathrm{p}<0.01$ vs C- siRNA. (C) ELISA analysis of VEGF release in supernatant by c-siRNA and FLNA siRNA H727 cells. All data are expressed as mean \pm SD of three independent experiments. $* *=p<0.01, * *=p<0.01$ vs C- siRNA.

A)

IP FLNA:

Rap1

FLNA
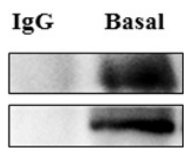

B)
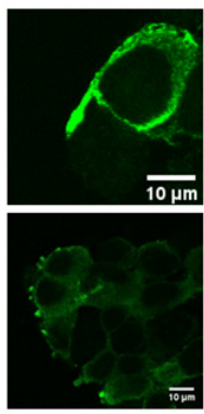

FLNA
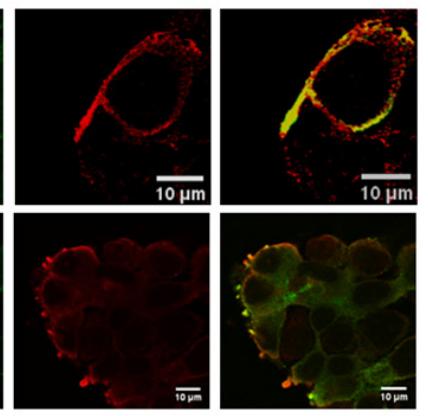

Rap1

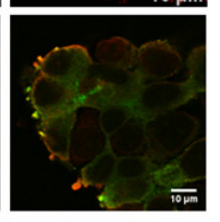

MERGE

C)

C-siRNA FLNA siRNA

Rap1 (21 kDa)

GAPDH (36 kDa)
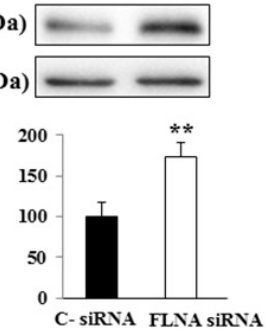

Figure 4: (A) Representative Immunoblot image shows thatFLNA interacts with Rap1. H727 lysate was immunoprecipitated with FLNA antibody or normal mouse IgG and the presence of Rap1 was detected using anti-Rap1 antibody. The presence of equal amounts of FLNA in the immunoprecipitates was confirmed by stripping and reprobing with anti-FLNA. As negative control, normal mouse IgG was used for the immunoprecipitation (IP). (B) Representative confocal microscopy images of fixed H727 cells stained for FLNA (green) and Rap 1 (red). The results shown are representative images of three individual experiments. Scale bar $10 \mu \mathrm{m}$. (C) Representative immunoblots of Rap1 expression in H727 cells transfected with C-siRNA or FLNA siRNA. The graph shows the ratio of Rap1/GAPDH normalized to $\mathrm{C}$-siRNA (mean value \pm SD from 3 independent experiments). $* *=p<0.01 \mathrm{vs} C$ - siRNA. 


\section{FLNA interacts with Rap1 in $\mathrm{H} 727$ cells}

Since Rap1 small GTPase is involved in the control of cell migration and adhesion and it plays a key role in neuroendocrine tumors [16], we hypothesized that the effects of FLNA could be mediated, at least in part, by a modulation of Rap1 expression. To examine this hypothesis, we evaluated the possible interaction between FLNA and Rap1 by immunoprecipitation. As shown in Figure 4A, Rap1 interacts with FLNA in H727 cells. This result was confirmed by immunofluorescence analysis, in which FLNA co-localized with Rap1 and interestingly, they co-localized mainly in cellular protrusions (Figure 4B). Interestingly, FLNA silencing increased Rap1 expression levels in $\mathrm{H} 727$ cell with respect to control cells ( $+73 \pm 18 \%, p<0.01$ vs $\mathrm{C}$-siRNA) (Figure $4 \mathrm{C})$, thus suggesting that FLNA is involved in the regulation of Rap1 expression.

\section{Implication of Rap1 in FLNA effect on cell proliferation, cell adhesion and cell migration}

To characterize the mechanism by which FLNA affects cell proliferation in $\mathrm{H} 727$ cells, we co-transfected Rap1 siRNA and FLNA siRNA with the aim of blocking the over-expression of Rap1 induced by FLNA silencing. FLNA silencing strongly reduced cyclin D1 expression and cell proliferation in $\mathrm{H} 727$ cells $(-61 \pm 2 \% \mathrm{p}<0.05$ vs

A)

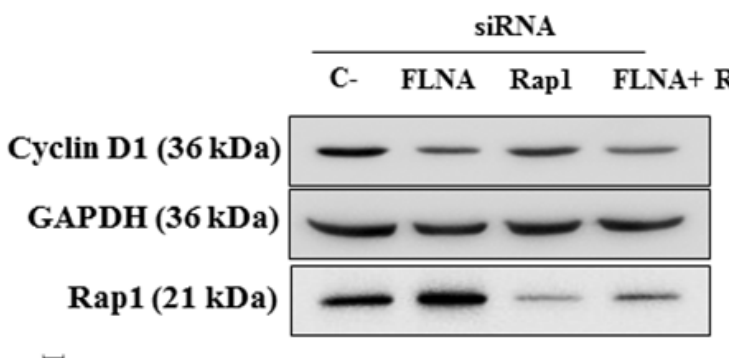

C)

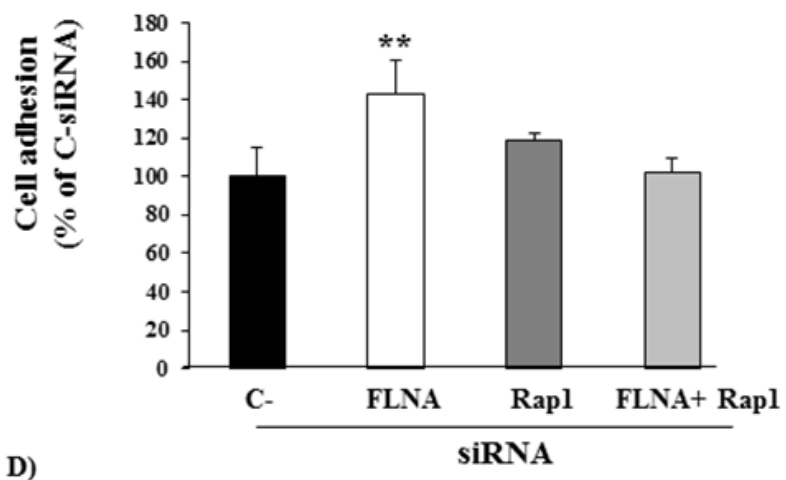

B)
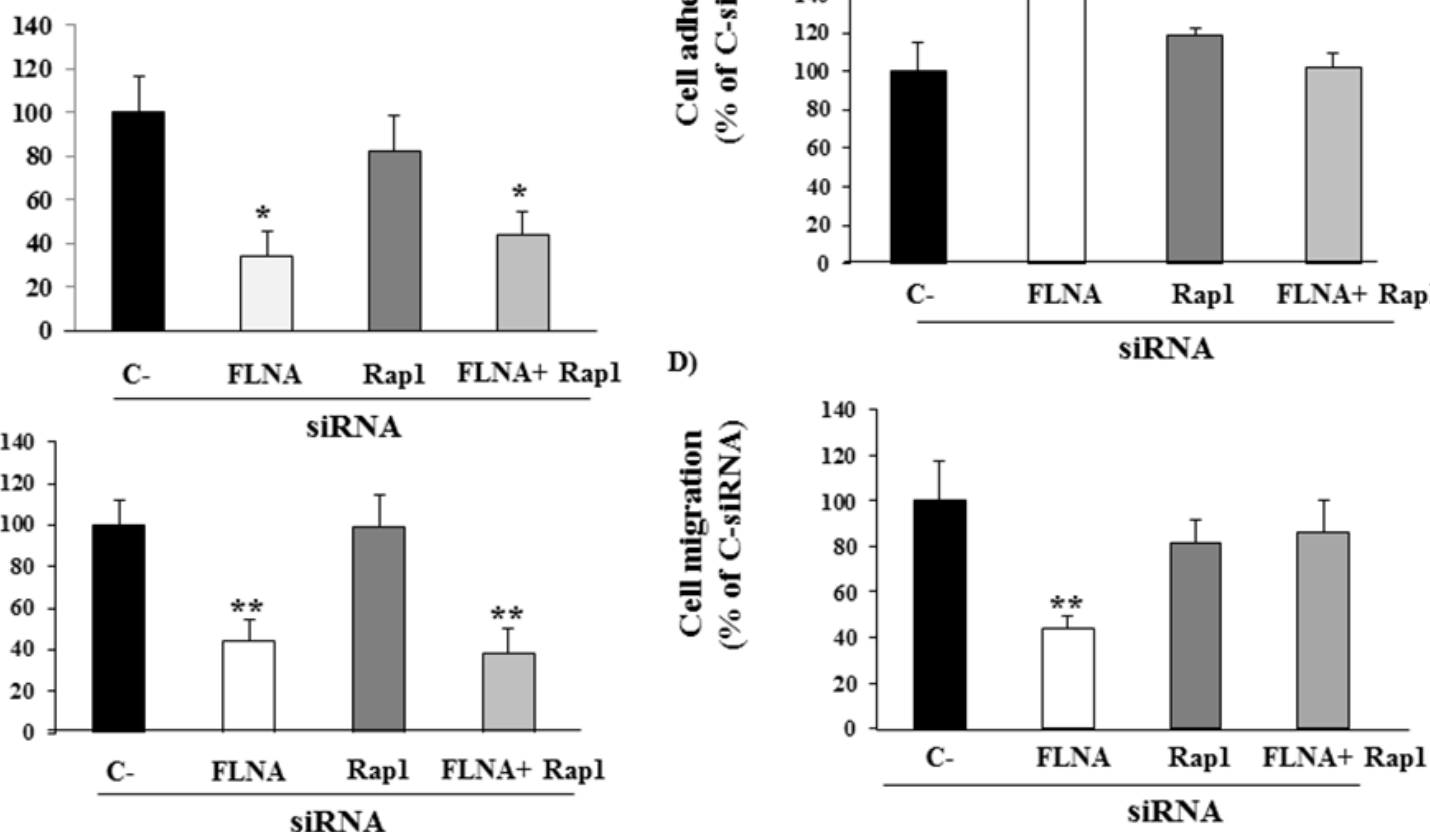

Figure 5: FLNA effect on cell proliferation, adhesion and cell migration and Rap1 involvement. (A) FLNA siRNA-treated cells showed a strong decrease in Cyclin D1 expression and cell proliferation in H727 cells not via Rap1. H727 cells were transfected with control siRNA, FLNA siRNA alone or together with Rap1 siRNA. The graph shows the quantification of Cyclin D1 normalized to GAPDH. Data represent mean \pm SD of three independent experiments. One-way ANOVA; Bonferroni post test $*=p<0.05$ vs C- siRNA. (B) The graph shows BrdU incorporation during DNA synthesis in H727 cells transfected with C-siRNA, FLNA siRNA alone or together with Rap1 siRNA. Data represent mean \pm SD of three independent experiments. One-way ANOVA; Bonferroni post test $* *=p<0.01$ vs C- siRNA. (C) H727 cells transfected with C-siRNA or FLNA siRNA alone or together with Rap1 siRNA were incubated in complete medium for 90 min at $37^{\circ} \mathrm{C}$. Experiments carried out in $\mathrm{H} 727$ cells were repeated at least 4 times. The graph shows the quantification of adherent cells. Values represent mean $( \pm \mathrm{SD})$. One-way ANOVA; Bonferroni post test $* *=\mathrm{p}<0.01$ vs C-siRNA. (D) H727 cells transfected with C-siRNA, FLNA siRNA alone or together with Rap1 were incubated for $24 \mathrm{~h}$ with serum-free medium. Migratory cells were quantified by fluorometric plate reader. Experiments were performed in triplicate 3 times. Values represent mean $( \pm \mathrm{SD})$; One-way ANOVA; Bonferroni post test $* *=\mathrm{p}<0.01$ vs C-siRNA. 
C-siRNA and $-56 \pm 11 \mathrm{p}<0.01$, respectively), confirming previous results in PNT cells. However, Rap1 knock down did not prevent the effects on cell proliferation of suppressing FLNA expression (Figure 5A, 5B). This result suggests that FLNA promotes cell proliferation in $\mathrm{H} 727$ cells, but not via Rap1.

Conversely, FLNA silencing increased cell adhesion $(+43 \% \pm 18 \%, \mathrm{p}<0.01$ vs $\mathrm{C}-\mathrm{siRNA})$ and decreased cell migration $(-56 \pm 7 \%, \mathrm{p}<0.01$ vs $\mathrm{C}$-siRNA). Interestingly, Rap1 silencing prevented the effects on adhesion and migration of suppressing FLNA expression in $\mathrm{H} 727$ cells (Figure 5C, 5D). These data suggest that FLNA effects on cell adhesion and migration are mediated by Rap1

\section{DISCUSSION}

Cytoskeleton protein FLNA appears as a crucial element in PNTs cells as demonstrated in various types of cancers $[5,6]$, in which FLNA expression is correlated with the cancer metastatic potential.

In this study, we first found that FLNA is expressed in typical PNT samples and in the appropriate cellular model H727 cell line. In this respect, multiple studies detected FLNA over-expression in human glioblastomas [17], in pancreatic cancer [18], salivary gland adenoid cystic carcinoma [19], in peripheral cholangiocarcinomas [20] and many others.

We also showed that FLNA expression increased progressively with malignant grade, as reported in several studies, in which FLNA expression is correlated with cancer aggressiveness [21]. In this respect, circulating FLNA has been detected in plasma samples obtained from breast cancer patients using a monoclonal antibody, while no FLNA was found in plasma from normal subjects [22].

Next, the associations between FLNA expression and the clinicopathological features of the patients with PNT were investigated. Interestingly, FLNA expression is significantly correlated with clinical parameters, including age, gender, $\mathrm{T}$ and $\mathrm{N}$ staging.

Thus, the increase of FLNA expression in malignant PNTs, together with the correlation between its expression and additional histopathologic characteristics suggests that FLNA may be a useful tool for individualized therapeutic strategy and its expression may be considered as a potential diagnostic marker.

The knockdown of FLNA was associated with a decrease in metastasis and proliferation in different cancer cells, including human melanoma cells [23]. In this work, we report that FLNA promotes PNT cell proliferation and colony formation, crucial mechanisms involved in cancer progression. In particular, we demonstrated that the cytostatic effect of FLNA silencing is consistent with the inhibition of the cell cycle at the G1/S transition in PNT cells, corresponding to a decrease in cyclin D1 expression. These results confirmed the central role of FLNA in tumorigenesis.
Recently, the involvement of the cytoskeletal actinbinding proteins in angiogenesis has been suggested as a target for anti-neovascular cancer therapy in vitro. In particular, a positive relationship between FLNA and VEGF has been demonstrated in patients with lung cancer [14], suggesting that FLNA is implicated in the control of angiogenesis through links with VEGF. Interestingly, overexpression of VEGF, together with VEGF receptors, has been observed in PNTs, suggesting that an autocrine activation of VEGF pathway may be involved in PNT tumorigenesis and progression $[2,24]$. Consistently with this observation, we demonstrated that FLNA silencing significantly decreased VEGF expression in PNT cells. Moreover, VEGF release was drastically reduced in $\mathrm{H} 727$ cells silenced for FLNA, suggesting that FLNA silencing also suppressed VEGF secretion. These data support the key role of FLNA in promoting angiogenesis.

Increasing evidence demonstrated that FLNA is essential for the regulation of cell migration and adhesion $[25,26]$. In this respect, FLNA is required for podosome stabilization, extracellular matrix degradation and three-dimensional mesenchymal migration [27]. In our neuroendocrine model, we demonstrated that FLNA knockdown decreased cell migration and increased cell adhesion.

Due to its functions in the control of cell mobility, cell adhesion, cell proliferation, angiogenesis and colony formation, it is reasonable that FLNA may represent a biomarker for PNT diagnosis and prediction of clinical outcomes.

By virtue of its scaffolding function, FLNA also interacts with more than 90 functionally different proteins involved in tumorigenesis and metastasis [8, 10, 23]. In fact, FLNA anchors GTPase signaling proteins and coordinates their actin-remodeling activities, leading the formation of lamellipodia and filopodia [11]. In addition to the multiple proteins binding FLNA, we found that FLNA interacts with a novel downstream effector Rap1 small GTPase, and interestingly, these proteins colocalizes in cellular protrusions. In fact, Rap1 is involved in the control of cell migration and adhesion by enhancing integrin-mediated cell--matrix attachment in several cell types [12].

Mechanistically, we showed that FLNA downregulates the expression of Rap1 in H727 cells. Since Rap1 is also involved in regulating cell adhesion and proliferation in PNT cells [16], we speculated that down-regulation of Rap1 expression by FLNA modulates these cellular activities, essential for cancer progression and metastasis development. Considering that FLNA knockdown increased Rap1 expression, we evaluated whether or not Rap1 over-expression silencing was able to abolish the effects of FLNA on cell proliferation, cell adhesion and migration. We concluded that FLNA increased cell migration and decreased cell adhesion by a Rap1-dependent pathway, but it increased cell proliferation via a Rap1independent mechanism. 
These results underline the crucial role of FLNA in cancer progression and aggressiveness and consequently its potential interest in PNTs therapy. In particular, the involvement of FLNA pathway in mediating PNT progression might not only provide valuable diagnostic and prognostic markers, but also novel therapeutic targets.

\section{MATERIALS AND METHODS}

\section{Pulmonary neuroendocrine tumor cell cultures}

The study was approved by the local ethics committee. Informed consent was obtained from all subjects involved in the study. Human neuroendocrine cells were obtained from 5 typical PNTs that were enzymatically dissociated in DMEM containing $2 \mathrm{mg} /$ $\mathrm{mL}$ collagenase (Sigma-Aldrich CorporateHeadquarters St. Louis, MO) at $37^{\circ} \mathrm{C}$ for $2 \mathrm{~h}$. Dispersed cells were cultured in DMEM supplemented with $10 \%$ fetal bovine serum, $2 \mathrm{mM}$ glutamine and antibiotics. Human typical bronchial carcinoid cell line $\mathrm{H} 727$ were purchased from ATCC in 2013. Cells were grown in RPMI 1640 medium supplemented with $10 \% \mathrm{FBS}, 2 \mathrm{mM}$ glutamine and antibiotics. $\mathrm{H} 727$ cell line was cultured at $37^{\circ} \mathrm{C}$ in $5 \% \mathrm{CO}_{2}$ atmosphere. $\mathrm{H} 727$ were tested for mycoplasma contamination by using N-GARDE mycoplasma PCR reagent set (\#EMK090020, Euroclone).

\section{Immunohistochemistry}

Immunohistochemistry experiments were performed on sections from 66 PNTs retrieved from the archives of Pathology Unit of IRCCS Ospedale Maggiore Policlinico, Milan, Italy. After dewaxing in Bioclear and rehydrating in ethanol, the sections were pretreated in a water bath set to $98^{\circ} \mathrm{C}$ in $0.01 \mathrm{M}$ citrate buffer for 25 minutes. FLNA antibody (MAB1678, Millipore, 1:600 diluition) was used, and antigen-antibody detection was performed with the MACH1 universal polymer detection kit (Biocare Medical). The evaluation of immunohistochemical staining was blind and FLNA immunoreactivities were graded according to an immunohistochemical score as previously described [28].

\section{FLNA and Rap1 silencing in pulmonary neuroendocrine cells}

Gene silencing was performed in PNTs and H727 cells using species-specific human FLNA pre-designed siRNA (\#4392420, Invitrogen) and Rap1 siRNA (\#sc36384 Santa Cruz), using Lipofectamine 2000 transfection reagent (\#11668-019, Invitrogen) according to the manufacturer's instruction, for $72 \mathrm{~h}$ and $48 \mathrm{~h}$ of incubation, respectively.

In order to obtain the best efficiency of FLNA silencing three different human FLNA silencer select predesigned siRNAs were tested.
Preliminary experiments to determine the optimal concentration of siRNAs and the kinetics of silencing of FLNA and Rap1 were performed. A negative control siRNA (C- siRNA) (AM4611, Invitrogen), a non-targeting sequence without significant homology to the sequence of human, mouse or rat transcripts, was used in each experiment. Western blotting was performed in each experiment to control the expression level of FLNA and Rap1 in silenced cells.

\section{Proliferation assay}

Cell proliferation was assessed by colorimetric measurement of 5-bromo-2'-deoxyuridine (BrdU) incorporation during DNA synthesis in proliferating cells, according to the instruction of the manufacturer, as previously reported [29]. Briefly, after transfection with FLNA siRNA, Rap1 siRNA or Rap1 together with FLNA siRNAs, cells were incubated with complete medium for $24 \mathrm{~h}$ hours and then with BrdU for $2 \mathrm{~h}$ (cell lines) and $24 \mathrm{~h}$ (primary cultures) to allow BrdU incorporation in newly synthesized cellular DNA. All experiments were repeated at least three times and each determination was done in triplicate.

\section{Colony formation}

After FLNA silencing, $\mathrm{H} 727$ cells were seeded into 6-well plates and 96-well plates in triplicate at a density of 1000 cells or 250 cells, respectively, and incubated in a $37^{\circ} \mathrm{C}$ for 7 days. To visualize colonies, we fixed the cells for 10 min with 4\% PFA and then we stained cell clones for $30 \mathrm{~min}$ with a solution containing $0.05 \%$ crystal violet, followed by water rinses to remove excess dye. The dishes were then photographed.

To obtain number of cells, we incubated cells with cell quantification solution, according to the manufacturer's protocol (\#ECM570, Millipore), for 4 hours at $37^{\circ} \mathrm{C}$ and then, we measured absorbance at $490 \mathrm{~nm}$. Images of H727 clones were acquired using brightfield microscopy with a $4 \mathrm{X}$ objective lens (Widefield IX53 Inverted, Olympus). Average of colony area is measured using the NIH software ImageJ.

\section{Cell cycle analysis}

Cells were cultured in 6 well plates $\left(6 \times 10^{5}\right.$ cells per well) with $1 \mathrm{ml}$ of medium. After $72 \mathrm{~h}$ of silencing fresh medium was added to perform the cell cycle analysis and left for 6 hours. Cells were harvested and washed twice in PBS $1 \mathrm{X}$ at $4^{\circ}$. Then cells were fix in $1 \mathrm{ml}$ of cold $80 \%$ ethanol adding drop wise to the pellet while vortexing and incubated at $4^{\circ}$ for $30 \mathrm{~min}$. After the cells were centrifuge at 2000rpm for $5 \mathrm{~min}$ to remove ethanol and then washed twice in PBS $1 \mathrm{X}$ at $4^{\circ}$. Finally the cells were stained for 30 minutes in DAPI solution (PBS, 0.01\% TRITON X-100, $1 \mathrm{ug} / \mathrm{ml}$ DAPI-Invitrogen ${ }^{\mathrm{TM}}$ ) at RT and then DNA content 
was read at BD FACSymphony A5. The results were analyzed using FlowJo ${ }^{\circledR}$ software (FlowJo LLC).

\section{Immunoprecipitation of Rap1}

H727 cells were lysed in lysis buffer in the presence of protease inhibitors. The homogenates were centrifuged for $10 \mathrm{~min}$ at $14,000 \mathrm{rpm}$ at $4^{\circ} \mathrm{C}$ and the supernatant incubated with $2 \mu \mathrm{g}$ of FLNA antibody (H00002316-M01, Abnova, $1: 1000$ ) and mixed overnight on a rotating wheel at $4^{\circ} \mathrm{C}$.

Then $20 \mu \mathrm{l}$ of resuspended volume of Protein A/G PLUS-Agarose beads (Santa Cruz) were added to the mix antibody-lysate and incubated at $4{ }^{\circ} \mathrm{C}$ on a rotating device for 3 hours. Immunoprecipitates were collected by centrifugation at 2,500 rpm for 5 minutes at $4{ }^{\circ} \mathrm{C}$. The pellet was washed 5 times with lysis buffer, each time repeating the centrifugation step above. After the final wash, pellet was resuspended in SDS sample buffer containing reducing agents and analyzed by Western blotting, to visualize Rap1 (07916, Millipore). The presence of equal amounts of receptor in the immunoprecipitates was confirmed by stripping and reprobing with anti-FLNA antibody. As a negative control, $2 \mu \mathrm{g}$ of normal mouse IgG (\#sc2025, negative control, Santa Cruz) was used for the immunoprecipitation (IP).

\section{Fluorescence microscopy}

H727 cells were fixed with 4\% paraformaldehyde for 10 minutes, washed several times in PBS and treated with $0.1 \%$ Triton X-100 buffer. After blocking with $2 \%$ BSA and 5\% NGS in PBS for $1 \mathrm{~h}$, cells were incubated with anti-FLNA (MAB1678, 1:200, Millipore) and antiRap1 (1:50, 07-916, Millipore) antibodies and then stained with Alexa Fluor 488-conjugated secondary antibody and AlexaFluor 546-conjugated secondary (1:1000, Invitrogen, Carlsbad, CA) for $1 \mathrm{~h}$ at room temperature.

Coverslips were mounted on glass slides with one drop of liquid mountant (ProLong ${ }^{\circledR}$ Gold Antifade Mountant, ThermoFisher). All images were collected using Olympus Fluoview FV1000 confocal microscope.

\section{Cyclin D1 and Rap1 western blotting}

To analyze cyclin D1 expression level, H727 cells were treated with C- siRNA, FLNA siRNA, or Rap1 siRNA together with FLNA siRNA, then serum starved for $24 \mathrm{~h}$ and incubated with complete medium for $6 \mathrm{~h}$ at $37^{\circ} \mathrm{C}$. Cells were then lysed in lysis buffer in the presence of protease inhibitors. Proteins were separated on SDS/ polyacrylamide gels and transferred to a nitrocellulose filter. A 1:1000 dilution of anti-cyclin D1 was used as primary antibody (ENT1173, Elabscience, Bethesda, MD) and an antirabbit horseradish peroxidase-linked antibody was used as secondary antibody. To analyze Rap1 expression level, H727 cells were treated with C-siRNA and FLNA
siRNA. Cells were then lysed in lysis buffer in the presence of protease inhibitors. Proteins were separated on SDS/ polyacrylamide gelsand transferred to a nitrocellulose filter. The 1:1000 dilution of anti-Rap1 and an antirabbit horseradish peroxidase- linked antibody were used. GAPDH (AM4300, Ambion, 1:2000) was used as housekeeping.

\section{VEGF protein expression}

To study VEGF expression, primary PNTs cell or H727 cells were transfected with FLNA siRNA or negative control siRNA for $72 \mathrm{~h}$ and incubated with complete medium for $24 \mathrm{~h}$ at $37^{\circ} \mathrm{C}$. Cells were then lysed in lysis buffer in the presence of protease inhibitors. Proteins were separated on SDS/polyacrylamide gels and transferred to a nitrocellulose filter. The 1:1000 dilution of anti-VEGF (ab46154, Abcam, UK) and an antirabbit horseradish peroxidase-linked antibody were used. GAPDH was used as housekeeping.

To analyse VEGF secretion, H727 cells were plated in 24-well tissue culture plates and incubated with FLNA siRNA or negative control siRNA for 72 h. After, fresh medium was added for $24 \mathrm{~h}$ and then supernatants were collected. Media were analyzed by a commercially available sandwich human VEGF ELISA kit (DuoSet ELISA, R\&D), according to the manufacturer's instructions. Assays were performed in triplicate for three independent experiments and the results were reported as picograms (pg) of VEGF per ml.

\section{Cell adhesion assay}

H727 cells silenced for FLNA siRNA or Rap1 siRNA together with FLNA siRNA were plated onto a collagen type IV-coated 48-well plate and incubated with complete medium for $90 \mathrm{~min}$ at $37^{\circ} \mathrm{C}$, as by manufacturer's protocol (CBA-061, Cell Biolabs INC). Briefly, nonadherent cells were removed by gently washing plates 4-5 times with PBS, adherent cells were lysed with lysis buffer and subsequently detected with CyQuant ${ }^{\mathbb{}}$ GR Dye (Cell Biolabs INC). Finally, each extracted sample was quantified by measuring fluorescence with a fluorescence plate reader at $480 \mathrm{~nm} / 520 \mathrm{~nm}$. The experiment was performed four times in quadruplicate.

\section{Cell migration assay}

H727 cells, transfected with negative control siRNA, FLNA siRNA or Rap1 together with FLNA siRNAs were plated in polycarbonate membrane plate (MDCBA-106, Cell Biolabs INC) in serum-free medium and placed into the feeder tray, containing chemoattractant solution, according to the instruction of the manufacturer. After 24 hours of incubation at $37^{\circ} \mathrm{C}$, migratory cells were first dissociated from the membrane, then stained and quantified after extraction using a fluorometric plate reader. 


\section{Statistical analysis}

The results were expressed as the mean $\pm \mathrm{SD}$. A paired two-tailed Student's test was used to detect the significance between two series of data. $\mathrm{P}<0.05$ was accepted as statistically significant.

One-way ANOVA analysis was used to compare three or more groups, followed by Bonferroni post-hoc test. Calculations were performed by GraphPad Prism 4.0 software (GraphPad Software, Inc., La Jolla, CA). P $<0.05$ was accepted as statistically significant.

\section{Abbreviations}

FLNA: filamin-A; PNT: pulmonary neuroendocrine tumor; HIF-1 $\alpha$ : hypoxia-inducible factors-1 $\alpha$; VEGF: vascular endothelial growth factor; siRNA: small interfering RNA; BrdU: 5-bromo-2'-deoxyuridine; IP: immunoprecipitation; GAPDH: Glyceraldehyde-3Phosphate Dehydrogenase.

\section{Author contributions} Lania.

Conception and design: Eleonora Vitali, Andrea

Development of methodology: Eleonora Vitali, Valeria Cambiaghi.

Acquisition of data: Eleonora Vitali, Pierluigi Novellis, Giulia Veronesi, Stefano Ferrero, Ilena Boemi, Lorenzo Rosso.

Analysis and interpretation of data (e.g., statistical analysis, biostatistics, computational analysis): Eleonora Vitali.

Writing, review, and/or revision of the manuscript: Eleonora Vitali, Giovanna Mantovani, Anna Spada, Alloisio Marco, Andrea Lania.

Administrative, technical, or material support (i.e., reporting or organizing data, constructing databases): Eleonora Vitali, Ilena Boemi, Lorenzo Rosso.

Study supervision: Andrea Lania.

\section{ACKNOWLEDGMENTS}

The authors are grateful to Dr. Sara Piccini for editorial assistance.

\section{CONFLICTS OF INTEREST}

The authors declare no potential conflicts of interest.

\section{GRANT SUPPORT}

This work has been supported by AIRC grant (AL, IG-13051) and Fellowship to E.V. and P. N. from Fondazione Umberto Veronesi.

\section{REFERENCES}

1. Travis WD. Pathology and diagnosis of neuroendocrine tumors: lung neuroendocrine. Thorac Surg Clin. 2014; 24: 257-66. https://doi.org/10.1016/j.thorsurg.2014.04.001.

2. Gridelli C, Rossi A, Airoma G, Bianco R, Costanzo R, Daniele B, Chiara GD, Grimaldi G, Irtelli L, Maione P, Morabito A, Piantedosi FV, Riccardi F. Treatment of pulmonary neuroendocrine tumours: state of the art and future developments. Cancer Treat Rev. 2013; 39: 466-72. https://doi.org/10.1016/j.ctrv.2012.06.012.

3. Noel-Savina E, Descourt R. Focus on treatment of lung carcinoid tumor. Onco Targets Ther. 2013; 6: 1533-7. https://doi.org/10.2147/OTT.S32464.

4. Jiang X, Yue J, Lu H, Campbell N, Yang Q, Lan S, Haffty BG, Yuan C, Shen Z. Inhibition of filamin-A reduces cancer metastatic potential. Int J Biol Sci. 2012; 9: 67-77. https:// doi.org/10.7150/ijbs.5577.

5. Savoy RM, Ghosh PM. The dual role of filamin A in cancer: can't live with (too much of) it, can't live without it. Endocr Relat Cancer. 2013; 20: R341-56. https://doi.org/10.1530/ ERC-13-0364.

6. Yue J, Huhn S, Shen Z. Complex roles of filamin-A mediated cytoskeleton network in cancer progression. Cell Biosci. 2013; 3: 7. https://doi.org/10.1186/2045-3701-3-7.

7. Djinovic-Carugo K, Carugo O. Structural portrait of filamin interaction mechanisms. Curr Protein Pept Sci. 2010; 11: 639-50. https://doi.org/CPPS-75.

8. Zhou AX, Hartwig JH, Akyürek LM. Filamins in cell signaling, transcription and organ development. Trends Cell Biol. 2010; 20: 113-23. https://doi.org/10.1016/j. tcb.2009.12.001.

9. Razinia Z, Mäkelä $T$, Ylänne $J$, Calderwood $D$ a. Filamins in mechanosensing and signaling. Annu Rev Biophys. 2012; 41: 227-46. https://doi.org/10.1146/ annurev-biophys-050511-102252.

10. Nakamura F, Stossel TP, Hartwig JH. The filamins: organizers of cell structure and function. Cell Adhes Migr. 2011; 5: 160-9. https://doi.org/10.4161/cam.5.2.14401.

11. Ohta Y, Suzuki N, Nakamura S, Hartwig JH, Stossel TP. The small GTPase RalA targets filamin to induce filopodia. Proc Natl Acad Sci U S A. 1999; 96: 2122-8. https://doi. org/10.1073/pnas.96.5.2122.

12. Caron E. Cellular functions of the Rap1 GTP-binding protein: a pattern emerges. J Cell Sci. 2003; 116: 435-40. https://doi.org/10.1242/jcs.00238.

13. Zheng X, Zhou AX, Rouhi P, Uramoto H, Borén J, Cao Y, Pereira T, Akyürek LM, Poellinger L. Hypoxia-induced and calpain-dependent cleavage of filamin A regulates the hypoxic response. Proc Natl Acad Sci U S A. 2014; 111: 2560-5. https://doi.org/10.1073/pnas.1320815111. 
14. Uramoto H, Akyürek LM, Hanagiri T. A positive relationship between filamin and VEGF in patients with lung cancer. Anticancer Res. 2010; 30: 3939-44.

15. Semenza GL. Hypoxia-inducible factors: mediators of cancer progression and targets for cancer therapy. Trends Pharmacol Sci. 2012; 33: 207-14. https://doi.org/10.1016/j. tips.2012.01.005.

16. Vitali E, Cambiaghi V, Spada A, Tresoldi A, Zerbi A, Peverelli E, Carnaghi C, Mantovani G, Lania AG. cAMP effects in neuroendocrine tumors: the role of Epac and PKA in cell proliferation and adhesion. Exp Cell Res. 2015; 339: 241-51. https://doi.org/10.1016/j.yexcr.2015.11.011.

17. Sun L, Hui AM, Su Q, Vortmeyer A, Kotliarov Y, Pastorino S, Passaniti A, Menon J, Walling J, Bailey R, Rosenblum M, Mikkelsen T, Fine HA. Neuronal and glioma-derived stem cell factor induces angiogenesis within the brain. Cancer Cell. 2006; 9: 287-300. https://doi.org/10.1016/j. ccr.2006.03.003.

18. Logsdon CD, Simeone DM, Binkley C, Arumugam T, Greenson JK, Giordano TJ, Misek DE, Hanash S. Molecular profiling of pancreatic adenocarcinoma and chronic pancreatitis identifies multiple genes differentially regulated in pancreatic cancer. Cancer Res. 2003; 63: 264957. https://doi.org/10.3322/canjclin.53.1.5.

19. Frierson HF, El-Naggar AK, Welsh JB, Sapinoso LM, Su AI, Cheng J, Saku T, Moskaluk CA, Hampton GM. Large scale molecular analysis identifies genes with altered expression in salivary adenoid cystic carcinoma. Am J Pathol. 2002; 161: 1315-23. https://doi.org/10.1016/ S0002-9440(10)64408-2.

20. Guedj N, Zhan Q, Perigny M, Rautou PE, Degos F, Belghiti J, Farges O, Bedossa P, Paradis V. Comparative protein expression profiles of hilar and peripheral hepatic cholangiocarcinomas. J Hepatol. 2009; 51: 93-101. https:// doi.org/10.1016/j.jhep.2009.03.017.

21. Uhlen MA. Human protein atlas for normal and cancer tissues based on antibody proteomics. Mol Cell Proteomics. 2005; 4: 1920-32. https://doi.org/10.1074/mcp. M500279-MCP200.

22. Browne KA, Johnstone RW, Jans DA, Trapani JA. Filamin (280-kDa actin-binding protein) is a caspase substrate and is also cleaved directly by the cytotoxic $\mathrm{T}$ lymphocyte protease granzyme B during apoptosis. J Biol Chem. 2000; 275: 39262-6. https://doi.org/10.1074/jbc. C000622200.

23. Zhang K, Zhu T, Gao D, Zhang Y, Zhao Q, Liu S, Su T, Bernier M, Zhao R. Filamin A expression correlates with proliferation and invasive properties of human metastatic melanoma tumors: implications for survival in patients. J Cancer Res Clin Oncol. 2014; 140: 1913-26. https:/doi. org/10.1007/s00432-014-1722-3.

24. La Rosa S, Uccella S, Finzi G, Albarello L, Sessa F, Capella C. Localization of vascular endothelial growth factor and its receptors in digestive endocrine tumors: correlation with microvessel density and clinicopathologic. Hum Pathol. 2003; 34: 18-27. https://doi.org/10.1053/ hupa.2003.56.

25. D'Addario M, Arora PD, Fan J, Ganss B, Ellen RP, McCulloch CA. Cytoprotection against mechanical forces delivered through $\beta 1$ integrins requires induction of Filamin A. J Biol Chem. 2001; 276: 31969-77. https://doi. org/10.1074/jbc.M102715200.

26. Meyer SC, Sanan DA, Fox JE. Role of actin-binding protein in insertion of adhesion receptors into the membrane. $\mathrm{J}$ Biol Chem. 1998; 273: 3013-20. https://doi.org/10.1074/ jbc.273.5.3013.

27. Guiet R, Vérollet C, Lamsoul I, Cougoule C, Poincloux R, Labrousse A, Calderwood DA, Glogauer M, Lutz PG, Maridonneau-Parini I. Macrophage mesenchymal migration requires podosome stabilization by Filamin A. J Biol Chem. 2012; 287: 13051-62. https://doi.org/10.1074/jbc. M111.307124.

28. Vitali E, Cambiaghi V, Zerbi A, Carnaghi C, Colombo P, Peverelli E, Spada A, Mantovani G, Lania AG. Filamin-A is required to mediate SST2 effects in pancreatic neuroendocrine tumours. Endocr Relat Cancer. 2016; 23: 181-90. https://doi.org/10.1530/ERC-15-0358.

29. Lania AG, Mantovani G, Ferrero S, Pellegrini C, Bondioni S, Peverelli E, Braidotti P, Locatelli M, Zavanone ML, Ferrante E, Bosari S, Beck-Peccoz P, Spada A. Proliferation of transformed somatotroph cells related to low or absent expression of protein kinase A regulatory subunit 1A protein. Cancer Res. 2004; 64: 9193-8. https://doi. org/10.1158/0008-5472.CAN-04-1847. 